\title{
EDITORIAL
}

\section{The plurality of optical singularities}

\section{Guest Editors}

\author{
Michael Berry \\ (Co-Director of NATO ARW) \\ $H$ H Wills Physics \\ Laboratory, University of \\ Bristol, UK
}

\section{Mark Dennis \\ H H Wills Physics \\ Laboratory, University of \\ Bristol, UK}

\author{
Marat Soskin \\ (Co-Director of NATO ARW) \\ Institute of Physics, National \\ Academy of Sciences of \\ Ukraine, Kiev, Ukraine
}

\begin{abstract}
This collection of papers arose from an Advanced Research Workshop on Singular Optics, held at the Bogolyubov Institute in Kiev, Ukraine, during 24-28 June 2003. The workshop was generously financed by NATO, with welcome additional support from Institute of Physics Publishing and the National Academy of Sciences of Ukraine. There had been two previous international meetings devoted to singular optics, in Crimea in 1997 and 2000, reflecting the strong involvement of former Soviet Union countries in this research. Awareness of singular optics is growing within the wider optics community, indicated by symposia on the subject at several general optics meetings.
\end{abstract}

As the papers demonstrate, the field of singular optics has reached maturity. Although the subject originated in an observation on ultrasound, it has been largely theory-driven until recently. Now, however, there is close contact between theory and experiment, and we speculate that this is one reason for its accelerated development.

To single out particular papers for mention here would be invidious, and since the papers speak for themselves it is not necessary to describe them all. Instead, we will confine ourselves to a brief description of the main areas included in singular optics, to illustrate the broad scope of the subject.

Optical vortices are lines of phase singularity: nodal lines where the intensity of the light, represented by a complex scalar field, vanishes. The subject has emerged from flatland, where the vortices are points characterized by topological charges, into the much richer world of vortex lines in three dimensions. By combining Laguerre-Gauss or Bessel beams, or reflecting light from plates with spiral steps, intricate arrangements can be generated, with vortices that are curved, looped, knotted, linked or braided.

With light whose state of polarization varies with position, different singularities occur, associated with the vector nature of light. These are also lines, on which the electric (or magnetic) polarization ellipse is purely circular ( $\mathrm{C}$ lines) or purely linear (L lines). The patterns of ellipse-fields are different for purely paraxial and fully three-dimensional fields.

White-light diffraction generates richly coloured vortices-the colours of dark light. The description of these chromatic effects, and also those associated with polarization singularities, leads to new applications of coherence theory.

For non-monochromatic light, it is natural to seek singularities of the full electromagnetic field, rather than of the electric or magnetic field separately. Such electromagnetic singularities are the Riemann-Silberstein vortices; these are relativistically covariant nodal lines of a complex scalar field constructed from the electromagnetic field.

Optical fields have dynamical aspects, particularly those associated with angular momentum. Although angular momentum is not inevitably associated with optical singularities, in practice the two phenomena can occur together. Orbital angular momentum is associated with the spatial structure of light, and in beams with optical vortices it can be used to rotate particles in the field. Spin angular momentum is associated with the polarization structure of the light. There are tricky questions associated with the angular momentum of light in a refracting medium, echoing the Abraham-Minkowski controversy about linear momentum. 
In optically nonlinear materials (leading to second-harmonic generation, for example), new classes of phenomena can occur, such as, for example, dynamical interaction between vortex lines, whose stability needs to be considered.

At a more fundamental level, it is important to investigate quantum effects associated with optical singularities, and a start has been made. The dark centre of an optical vortex can be regarded as a window onto the vacuum fluctuations of quantum optics, with the quantum core emerging as a distinct entity when the classical light is intense. And for light in a rapidly and inhomogeneously flowing material, horizons can develop, analogous to those surrounding black holes in general relativity, and these new optical singularities can be regarded as wave catastrophes, and new associated quantum effects anticipated.

Three decades after wave dislocations were introduced as 'a new concept in ... wave theory', these phase singularities have been extensively explored and are now familiar. New ideas - in addition to those described in this special issue-continue to emerge. For example, $x$-ray vortices were observed recently; there is a proposal to create lenses to form atomic beams containing vortices; and astrophysical applications have been suggested for both photon orbital angular momentum and optical vortices. We can safely assume that the science of wave singularities will develop further, and diffuse into new areas of physics. 